\title{
AN ACH METHOD FOR SOLVING THE PERIODICITY PROBLEM OF A CLASS OF DIFFERENTIAL EQUATIONS
}

\section{MENGLONG SU and GENGHUA JIA}

Mathematics Science College

Luoyang Normal University

Luoyang 471934

P. R. China

e-mail: mlsulynu@163.com

Department of Mathematics and Physics

Luoyang Institute of Science and Technology

Luoyang 471023

P. R. China

\begin{abstract}
In this paper, using the idea of the aggregate function method, an $\mathrm{ACH}$ method is proposed to solve the periodicity problem of a class of differential equations. Under suitable conditions, we give a constructive proof the existence of the periodic solutions. Following the solution path, we can find the periodic solution via the reduced predictor-corrector algorithm by the ACH method.
\end{abstract}

2010 Mathematics Subject Classification: $65 \mathrm{H} 10$.

Keywords and phrases: ACH method, constructive proof, periodic solutions.

This work was supported by National Nature Science Foundation of China (No. 11671188).

Received July 31, 2018; Revised August 20, 2018

(C) 2018 Scientific Advances Publishers 


\section{Introduction}

As is well known, the periodicity problem plays a central role in the qualitative theory of differential equations for its significance in the physical sciences. Hence finding periodic solutions of ordinary differential equations is naturally an attracting topic. This paper deals with the problems of finding periodic solutions for vector ordinary differential equations of the form:

$$
x^{\prime}=f(t, x)=f(t+T, x)
$$

where $T$ is a fixed positive number. In [1], we used a homotopy interior point method to find the periodic solutions of (1.1). In [2], we applied proper perturbations to the equality constraints and hence enlarge the scope of choice of initial points. This point can improve the computational efficiency of the algorithm. However, the algorithms in [1, 2] become inefficient when the number of constraint functions increases largely. To overcome the difficulties mentioned above, we combine the aggregate function method [3,4] with the homotopy method [5-9] to develop an $\mathrm{ACH}$ method to solve problem (1.1). Compared with the algorithms in [1, $2]$, the $\mathrm{ACH}$ method can reduce the dimensions of the linear systems greatly, so this method can deal with problem (1.1) when the number of constraint functions increases largely. Under suitable conditions, the existence of a smooth path from a given interior point of the feasible region to the periodic solution point of problem (1.1) will be proven. Following this solution path, we can give an implementable globally convergent algorithm. Using the ACH method, we are able to solve problem (1.1) and thus improve the computational efficiency of the algorithm in [1, 2] greatly. 


\section{Main Results}

In this section, let $u_{i}(x), i=1, \ldots, m$ be $C^{3}$ functions, then let $X=\left\{x \in R^{n}: u_{i}(x) \leq 0, i=1, \ldots, m\right\}, X^{0}=\left\{x \in R^{n}: u_{i}(x)<0, i=1\right.$, $\ldots, m\}$ and $\partial X=X \backslash X^{0}$ be the boundary set of $X$. In addition, denote the nonnegative and positive orthants $R^{1}$ by $R_{+}^{1}$ and $R_{++}^{1}$, respectively. For all $x \in \partial X$, denote the active index set by $I(x)=\{i \in\{1, \ldots, m\}$ : $\left.u_{i}(x)=0\right\}$.

By introducing $C^{2}$ functions $\alpha_{i}(x) \in R^{n}, i=1, \ldots, m$, we need the following conditions which are often used in practice:

$\left(\mathrm{C}_{1}\right) X^{0}$ is nonempty and $X$ is bounded.

$\left(\mathrm{C}_{2}\right)$ For any $x \in \partial X$, if

$$
\sum_{i \in I(x)} \alpha_{i}(x) \beta_{i}=0, \beta_{i} \geq 0
$$

then $\beta_{i}=0, i \in I(x)$.

$\left(\mathrm{C}_{3}\right)$ There exists a closed subset $\hat{X} \subset X^{0}$ with nonempty interior $\hat{X}^{0}$, such that for any $x \in \partial X$, we obtain

$$
\left\{x+\sum_{i \in I(x)} \beta_{i} \alpha_{i}(x): \beta_{i} \geq 0 \text { for } i \in I(x) \text { and } \sum_{i \in I(x)} \beta_{i}>0\right\} \cap \hat{X}=\emptyset .
$$

In the following, we are devoted to obtaining the global convergence of the ACH method under conditions $\left(\mathrm{C}_{1}\right)-\left(\mathrm{C}_{3}\right)$.

Lemma 2.1. Let $\bar{u}(x)=\max _{1 \leq i \leq m}\left\{u_{i}(x)\right\}, X^{\prime}=\left\{x \in R^{n}: \bar{u}(x) \leq 0\right\}$, then $X^{\prime}=X$.

The non-differentiability of $\bar{u}(x)$ prevents us to give a effective method to solve the periodicity problem. To overcome this difficulty, we use the technique of the aggregate function, and hence consider the 
aggregate form of the inequality constraint functions $u_{i}(x), i=1, \ldots, m$ as follows:

$$
u(\theta \lambda, x)=\theta \lambda \ln \sum_{i=1}^{m} \exp \left(u_{i}(x) / \theta \lambda\right)
$$

where $\theta \in(0,1]$.

Let $X(\theta \lambda)=\left\{x \in R^{n}: u(\theta \lambda, x) \leq 0\right\}, X^{0}(\theta \lambda)=\left\{x \in R^{n}: u(\theta \lambda, x)<0\right\}$ and $\partial X(\theta \lambda)=X(\theta \lambda) \backslash X^{0}(\theta \lambda)$. The following Lemmas 2.2-2.6 are easily to deduce.

Lemma 2.2. For a given $\theta \in(0,1]$, the following two conclusions hold:

(1) For any $\lambda \in(0,1], \bar{u}(x) \leq u(\theta \lambda, x) \leq \bar{u}(x)+\theta \lambda \ln m$.

(2) For $0<\lambda_{1} \leq \lambda_{2} \leq 1, u\left(\theta \lambda_{1}, x\right) \leq u\left(\theta \lambda_{2}, x\right)$, in addition, when $\lambda \rightarrow 0, u(\theta \lambda, x) \rightarrow \bar{u}(x)$.

Lemma 2.3. If $u_{i}(x), i=1, \ldots, m$ is $C^{3}$, then $u(\theta \lambda, x)$ is also $C^{3}$, and the gradient of $u(\theta \lambda, x)$ with respect to $x$ is

$$
\nabla_{x} u(\theta \lambda, x)=\sum_{i=1}^{m} y_{i}(\theta \lambda, x) \nabla u_{i}(x)
$$

where

$$
y_{i}(\theta \lambda, x)=\exp \left(u_{i}(x) / \theta \lambda\right) / \sum_{i=1}^{m} \exp \left(u_{i}(x) / \theta \lambda\right) .
$$

Lemma 2.4. $y_{i}(\theta \lambda, x), i=1, \ldots, m$ in (2.2) satisfies that

(1) $0 \leq y_{i}(\theta \lambda, x)$ and $\sum_{i=1}^{m} y_{i}(\theta \lambda, x)=1$.

(2) $y_{i}(\theta \lambda, x) \rightarrow 0$ as $\lambda \rightarrow 0^{+}$and $X^{0}$ э $x \rightarrow \bar{x} \in \partial X$ for $i \notin I(\bar{x})$. 
Lemma 2.5. Under condition $\left(\mathrm{C}_{1}\right)$, we obtain:

(1) For any $\theta \in(0,1]$, and $\lambda \in(0,1], X(\theta \lambda) \subset X$.

(2) For any closed subset $N \subset X^{0}$, there exists a $\theta \in(0,1]$, such that $N \subset X^{0}(\theta)$

Set $\alpha(\theta \lambda, x)=\sum_{i=1}^{m} y_{i}(\theta \lambda, x) \alpha_{i}(x)$, then the following lemma holds:

Lemma 2.6. Under conditions $\left(\mathrm{C}_{1}\right)-\left(\mathrm{C}_{3}\right)$, then the following two conclusions hold:

(1) There exists a $\theta \in(0,1]$, such that for any $\lambda \in(0,1]$ and $x \in \partial X(\theta \lambda)$, if

$$
\alpha(\theta \lambda, x) \beta=0, \beta \geq 0,
$$

then $\beta=0$.

(2) For any closed subset $N \subset \hat{X}$, there exists a $\theta \in(0,1]$, such that for any $\lambda \in(0,1]$,

$$
\{x+y \alpha(\theta \lambda, x): y>0\} \cap N=\emptyset,
$$

where $x \in \partial X(\theta \lambda)$.

Proof. (1) If not, then there exist sequences $\left\{\lambda_{k}\right\}_{k=1}^{\infty},\left\{x^{(k)}\right\}_{k=1}^{\infty}$, such that $\lambda_{k} \rightarrow 0, x^{(k)} \in \partial X\left(\theta \lambda_{k}\right)$ and

$$
\alpha\left(\theta \lambda_{k}, x^{(k)}\right)=\sum_{i=1}^{m} y_{i}\left(\theta \lambda_{k}, x^{(k)}\right) \alpha_{i}\left(x^{(k)}\right)=0 .
$$

By Lemmas 2.4 and 2.5, we conclude that

$$
0 \leq y_{i}\left(\theta \lambda_{k}, x^{(k)}\right), \quad \sum_{i=1}^{m} y_{i}\left(\theta \lambda_{k}, x^{(k)}\right)=1
$$


and $X\left(\theta \lambda_{k}\right)$ is bounded. Hence there exist subsequences $\left\{\lambda_{k_{i}}\right\}_{i=1}^{\infty}$, $\left\{x^{\left(k_{i}\right)}\right\}_{i=1}^{\infty}$, and $\left\{y_{i}\left(\theta \lambda_{k_{i}}, x^{\left(k_{i}\right)}\right)\right\}_{i=1}^{\infty}$ such that $\lambda_{k_{i}} \rightarrow 0, x^{\left(k_{i}\right)} \rightarrow x^{*} \in \partial X$, and $y_{i}\left(\theta \lambda_{k_{i}}, x^{\left(k_{i}\right)}\right) \rightarrow y_{i}\left(0, x^{*}\right)$. Moreover, by Lemma 2.4, $y_{i}\left(0, x^{*}\right)=0$ for $i \notin I\left(x^{*}\right)$. Therefore when $k_{i} \rightarrow \infty$, from (2.4) and (2.5), we obtain

$$
\alpha\left(\theta \lambda_{k}, x^{(k)}\right) \rightarrow \sum_{i \in I\left(x^{*}\right)} y_{i}\left(0, x^{*}\right) \alpha_{i}\left(x^{*}\right)=0,
$$

and

$$
y_{i}\left(0, x^{*}\right) \geq 0, \sum_{i \in I\left(x^{*}\right)} y_{i}\left(0, x^{*}\right)=1,
$$

which contradicts condition $\left(\mathrm{C}_{2}\right)$.

(2) If not, then there exist sequences $\left\{\lambda_{k}\right\}_{k=1}^{\infty},\left\{\hat{x}^{(k)}\right\}_{k=1}^{\infty},\left\{\tilde{x}^{(k)}\right\}_{k=1}^{\infty}$, and $\left\{\widetilde{z}^{(k)}\right\}_{k=1}^{\infty}$ such that $\lambda_{k} \rightarrow 0, \hat{x}^{(k)} \in N, \tilde{x}^{(k)} \in \partial \Omega\left(\theta \mu_{k}\right), \widetilde{z}^{(k)}>0$, and

$$
\hat{x}^{(k)}=\tilde{x}^{(k)}+\alpha\left(\theta \lambda_{k}, \tilde{x}^{(k)}\right) \tilde{z}^{(k)}=\tilde{x}^{(k)}+\sum_{i=1}^{m} \alpha_{i}\left(\tilde{x}^{(k)}\right) y_{i}\left(\theta \lambda_{k}, \tilde{x}^{(k)}\right) \widetilde{z}^{(k)} .
$$

By Lemmas 2.4 and 2.5, we conclude that

$$
0 \leq y_{i}\left(\theta \lambda_{k}, \tilde{x}^{(k)}\right), \sum_{i=1}^{m} y_{i}\left(\theta \lambda_{k}, \tilde{x}^{(k)}\right)=1,
$$

and $X\left(\theta \lambda_{k}\right)$ is bounded. Hence there exist subsequences $\left\{\lambda_{k_{i}}\right\}_{i=1}^{\infty}$, $\left\{\hat{x}^{\left(k_{i}\right)}\right\}_{i=1}^{\infty},\left\{\tilde{x}^{\left(k_{i}\right)}\right\}_{i=1}^{\infty}$, and $\left\{y_{i}\left(\theta \lambda_{k_{i}}, \tilde{x}^{\left(k_{i}\right)}\right)\right\}_{i=1}^{\infty}$ such that $\lambda_{k_{i}} \rightarrow 0, \hat{x}^{\left(k_{i}\right)} \rightarrow$ $\hat{x} \in N \subset \hat{X}, \tilde{x}^{\left(k_{i}\right)} \rightarrow \tilde{x} \in \partial X$, and $y_{i}\left(\theta \lambda_{k_{i}}, \tilde{x}^{\left(k_{i}\right)}\right) \rightarrow y_{i}(0, \tilde{x})$. Moreover, 
by Lemma 2.4 , we have $y_{i}(0, \tilde{x})=0$ for $i \notin I(\tilde{x})$. So from (2.7), we obtain

$$
0 \leq y_{i}(0, \tilde{x}), \sum_{i \in I(\tilde{x})} y_{i}(0, \tilde{x})=1
$$

By (2.6) and condition $\left(\mathrm{C}_{2}\right)$, as $k_{i} \rightarrow \infty$, we have $\widetilde{z}^{\left(k_{i}\right)} \rightarrow \widetilde{z}$ and $0 \leq \widetilde{z}<\infty$. Then taking limits in (2.6), we have

$$
\hat{x}=\tilde{x}+\sum_{i \in B(\tilde{x})} y_{i}(0, \tilde{x}) \tilde{z} \alpha_{i}(\widetilde{x}),
$$

which contradicts condition $\left(\mathrm{C}_{3}\right)$.

For finding the periodic solution of the differential equations, we construct the following aggregate homotopy:

$$
H\left(P, P^{(0)}, \lambda\right)=\left(\begin{array}{c}
(1-\lambda)(x-x(T, x)+\alpha(\theta \lambda, x) y)+\lambda\left(x-x^{(0)}\right) \\
y u(\theta \lambda, x)-\lambda y^{(0)} u\left(\theta, x^{(0)}\right)
\end{array}\right)=0
$$

where $P=(x, y) \in R^{n} \times R^{1}$ and $P^{(0)}=\left(x^{(0)}, y^{(0)}\right) \in \hat{X}^{0} \times R_{++}^{1}$.

Given a point $P^{(0)}$, denote $H\left(P, P^{(0)}, \lambda\right)$ by $H_{P^{(0)}}(P, \lambda)$. Then let

$$
H_{P^{(0)}}^{-1}(0)=\left\{(P, \lambda) \in X(\theta \lambda) \times R_{+}^{1} \times(0,1]: H_{P^{(0)}}(P, \lambda)=0\right\}
$$

be the zero-point set of $H_{P^{(0)}}$.

The following theorem is our main result, which presents a theoretic base of the $\mathrm{ACH}$ method.

Theorem 2.1. Let $H$ be defined as in (2.8). In addition, let conditions $\left(\mathrm{C}_{1}\right)-\left(\mathrm{C}_{3}\right)$ hold, let $u_{i}(x), i=1, \cdots, m$ be $C^{3}$ functions and let $\alpha_{i}(x)$, $i=1, \cdots, m$ be $C^{2}$ mappings. Then the Equation (1.1) has a T-periodic 
solution in X. Moreover, for almost every $\left(x_{0}, y_{0}\right)$ there exists a $C^{1}$ path $(P(s), \lambda(s))$ of dimension 1 such that

$$
H\left(P^{(0)}, P(s), \lambda(s)\right)=0, \quad(P(0), \lambda(0))=\left(P^{(0)}, 1\right),
$$

and $\lambda(s)$ leads to 0 ; while $P(s)$ leads to a point $\left(x^{*}, y^{*}\right)$; in particular, $x\left(t, x^{*}\right)$ is a T-periodic solution of (1.1).

Proof. Let the Jacobi matrix of $H\left(P, P^{(0)}, \lambda\right)$ be $D H\left(P, P^{(0)}, \lambda\right)$, we obtain

$$
D H\left(P, P^{(0)}, \lambda\right)=\left(\frac{\partial H\left(P, P^{(0)}, \lambda\right)}{\partial P}, \frac{\partial H\left(P, P^{(0)}, \lambda\right)}{\partial P^{(0)}}, \frac{\partial H\left(P, P^{(0)}, \lambda\right)}{\partial \lambda}\right) .
$$

$\forall(P, \lambda) \in R^{n+1} \times(0,1]$, the matrix is

$$
\frac{\partial H\left(P, P^{(0)}, \lambda\right)}{\partial P^{(0)}}=\left(\begin{array}{cc}
-\lambda I & 0 \\
-\lambda y^{(0)} \nabla u\left(\theta, x^{(0)}\right)-\lambda u\left(\theta, x^{(0)}\right)
\end{array}\right) .
$$

$D H\left(P, P^{(0)}, \lambda\right)$ is of full row rank because $\partial H\left(P, P^{(0)}, \lambda\right) / \partial P^{(0)}$ is nonsingular, so 0 is a regular value of $H\left(P, P^{(0)}, \lambda\right)$. Then for almost all $P^{(0)}, 0$ is a regular value of $H_{P^{(0)}}$. So by the fact that $H\left(P, P^{(0)}, 1\right)=0$, we conclude that there exists a $C^{1}$ curve, which is denoted by $\left.\Gamma_{P^{(0)}}\right)$, such that

$$
H\left(P(s), P^{(0)}, \lambda(s)\right)=0, \quad(P(0), \lambda(0))=\left(P^{(0)}, 1\right) .
$$

Because

$$
\frac{\partial H_{P^{(0)}}\left(P^{(0)}, 1\right)}{\partial P}=\left(\begin{array}{cc}
I & 0 \\
y^{(0)} \nabla u\left(\theta, x^{(0)}\right) u\left(\theta, x^{(0)}\right)
\end{array}\right)
$$


is nonsingular, therefore by using the classification theorem of onedimensional smooth manifold, $\Gamma_{P^{(0)}}$ is diffeomorphic to the unit interval $(0,1]$.

Let $\left(P^{*}, \lambda^{*}\right)$ be a limit point of $\Gamma_{P^{(0)}}$. When $\lambda^{*}=1$, then from (2.8), we obtain

$$
\begin{aligned}
& x-x^{(0)}=0, \\
& y u(\theta, x)-y^{(0)} u\left(\theta, x^{(0)}\right)=0 .
\end{aligned}
$$

So $H\left(P, P^{(0)}, 1\right)=0$ has a unique solution $P=P^{(0)}$. Then the fact that $\left(P^{*}, \lambda^{*}\right)=\left(x^{*}, y^{*}, \lambda^{*}\right) \in X^{0}(1) \times R_{++}^{1} \times\{1\}$ does not occur.

Now we are devoting to proving the fact that $\left(P^{*}, \lambda^{*}\right)=\left(x^{*}, y^{*}, \lambda^{*}\right)$ $\in \partial\left(X\left(\lambda^{*}\right) \times R_{+}^{1}\right) \times(0,1]$ does not occur. If the conclusion does not hold, there exists a sequence of points $\left(x^{(k)}, y^{(k)}, \lambda k\right) \in \Gamma_{P^{(0)}}$ such that $x^{(k)} \rightarrow x^{*},\left\|y^{(k)}\right\| \rightarrow \infty$, and $\lambda_{k} \rightarrow \lambda^{*}$ as $k \rightarrow \infty$. From (2.8), we obtain

$$
\left(1-\lambda_{k}\right) \alpha\left(\theta \lambda_{k}, x^{(k)}\right) y^{(k)}+x^{(k)}-x^{(0)}=\left(1-\lambda_{k}\right)\left(x\left(T, x^{(k)}\right)-x^{(0)}\right) .
$$

As $k \rightarrow \infty$, the following equation holds:

$$
\left(\lim _{k \rightarrow \infty}\left(1-\lambda_{k}\right) y^{(k)}\right) \alpha\left(\theta, x^{*}\right)+x^{*}=x^{(0)} .
$$

Condition $\left(\mathrm{C}_{2}\right)$ and (2.10) yield that

$$
\lim _{k \rightarrow \infty}\left(1-\lambda_{k}\right) y^{(k)}=\beta^{*},
$$

where $\beta^{*} \geq 0$. So (2.10) and (2.11) yield that

$$
x^{*}+\alpha\left(\theta, x^{*}\right) \beta^{*}=x^{(0)},
$$

which contradicts Lemma 2.6. 
If $\lambda^{*}<1$, then (2.9) becomes

$$
\begin{aligned}
& \left(1-\lambda_{k}\right) \alpha\left(\theta \lambda_{k}, x^{(k)}\right) y^{(k)} \\
& \quad=\left(1-\lambda_{k}\right)\left(x^{(k)}-x\left(T, x^{(k)}\right)\right)-\lambda_{k}\left(x^{(k)}-x^{(0)}\right) .
\end{aligned}
$$

When $k \rightarrow \infty$, it is easy to show that the right-hand side of (2.13) is bounded and the left-hand side of (2.13) is infinite,. This results in a contradiction.

As a result, the conclusion that $\left(P^{*}, \lambda^{*}\right)=\left(x^{*}, y^{*}, \lambda^{*}\right) \in X \times R_{+}^{m} \times\{0\}$ holds. In the following, it is easy to show that $x\left(t, x^{*}\right)$ is a $T$-periodic solution of (1.1).

\section{References}

[1] M. L. Su, Existence of the periodic solutions of a class of periodicity problem of differential equations and related algorithms, Advances in Differential Equations and Control Processes 15(2) (2015), 83-92.

DOI: http://dx.doi.org/10.17654/ADECPMay2015_083_092

[2] M. L. Su and Q. Q. Liu, A constructive proof of existence of the periodic solutions of differential equations via a non-interior path algorithm, Far East Journal of Dynamical Systems 28(2) (2016), 89-99.

DOI: http://dx.doi.org/10.17654/DS028020089

[3] G. Kreisselmeier and R. Steinhauser, Systematic control design by optimizing a vector performance index, IFAC Proceedings Volumes 12(7) (1979), 113-117.

DOI: https://doi.org/10.1016/S1474-6670(17)65584-8

[4] D. Q. Mayne and E. Polak, Nondifferential optimization via adaptive smoothing, Journal of Optimization Theory and Applications 43(4) (1984), 601-613.

DOI: https://doi.org/10.1007/BF00935008

[5] R. B. Kellogg, T. Y. Li and J. A. Yorke, A constructive proof of the Brouwer fixedpoint theorem and computational results, SIAM Journal on Numerical Analysis 13(4) (1976), 473-483.

DOI: https://doi.org/10.1137/0713041 
[6] S. Smale, Global analysis and economics VI: Geometric analysis of Pareto optima and price equilibria under classical hypotheses, Journal of Mathematical Economics 3(1) (1976), 1-14.

DOI: https://doi.org/10.1016/0304-4068(76)90002-1

[7] S. N. Chow, J. Mallet-Paret and J. A. Yorke, Finding zeros of maps: Homotopy methods that are constructive with probability one, Mathematics of Computation 32(143) (1978), 887-899.

DOI: https://doi.org/10.1090/S0025-5718-1978-0492046-9

[8] Y. Li and Z. H. Lin, A constructive proof of the Poincaré-Birkhoff theorem, Transactions of the American Mathematical Society 347(6) (1995), 2111-2126.

DOI: https://doi.org/10.1090/S0002-9947-1995-1290734-4

[9] E. L. Allgower and K. Georg, Introduction to Numerical Continuation Methods, SIAM Society for Industry and Applied Mathematics, Philadelphia, New York, 2003.

DOI: https://doi.org/10.1137/1.9780898719154 